\title{
Mediterranean diet in pregnancy is protective for wheeze and atopy in childhood
}

\author{
L Chatzi, ${ }^{1}$ M Torrent, ${ }^{2}$ I Romieu, ${ }^{3}$ R Garcia-Esteban, ${ }^{4}$ C Ferrer, ${ }^{4}$ J Vioque, ${ }^{5}$ \\ M Kogevinas, ${ }^{1,4}$ J Sunyer ${ }^{4,6}$
}

\section{See Editorial, p 483}

- Supplementary table $\mathrm{S} 1$ is published online only at http:// thorax.bmj.com/content/vol63/ issue6

${ }^{1}$ Department of Social Medicine, Faculty of Medicine, University of Crete, Heraklion, Greece; ${ }^{2}$ Area de Salud de Menorca, IB-SALUT, Menorca, Spain; ${ }^{3}$ National Institute of Public Health, Cuernavaca,

Mexico; ${ }^{4}$ Centra de Recerca en Epidemiologia Ambiental

(CREAL), Institut Municipal d'Investigacio Medica,

Barcelona, Spain; ${ }^{5}$ Dpto Salud

Publica, Campus San Juan,

Universidad Miguel Hernandez,

Elche-Alicante, Spain;

${ }^{6}$ Universitat Pompeu Fabra,

Barcelona, Spain

Correspondence to:

Dr L Chatzi, Department of

Social Medicine, Faculty of

Medicine, University of Crete,

PO Box 2208, Heraklion, 71003,

Crete, Greece;

Ichatzi@med.uoc.gr

Received 22 March 2007

Accepted 4 September 2007

\begin{abstract}
Introduction: Dietary intake of specific nutrients or food groups during pregnancy could play a role in the risk of asthma and atopy in offspring, but specific dietary patterns have not been implicated. The authors evaluated the impact of maternal (during pregnancy) and child adherence to a Mediterranean diet on asthma and atopy in childhood.
\end{abstract}

Methods: Women presenting for antenatal care at all general practices in Menorca, a Mediterranean island in Spain, over a 12 month period starting in mid-1997 were recruited. 460 children were included in the analysis after 6.5 years of follow-up. Maternal dietary intake during pregnancy and children's dietary intake at age 6.5 years were assessed by food frequency questionnaires, and adherence to a Mediterranean diet was evaluated by a priori defined scores. During follow-up, parents completed questionnaires on the child's respiratory and allergic symptoms. Children underwent skin prick tests with six common aeroallergens.

Results: The prevalence rates of persistent wheeze, atopic wheeze and atopy at age 6.5 years were $13.2 \%$, $5.8 \%$ and $17.0 \%$, respectively. One-third (36.1\%) of mothers had a low quality Mediterranean diet during pregnancy according to the Mediterranean Diet Score, while the rest had a high score. A high Mediterranean Diet Score during pregnancy lat two levels, using "low" score as the reference) was found to be protective for persistent wheeze (OR 0.22; $95 \% \mathrm{Cl} 0.08$ to 0.58 ), atopic wheeze (OR 0.30; $95 \% \mathrm{Cl} 0.10$ to 0.90 ) and atopy (OR $0.55 ; 95 \% \mathrm{Cl} 0.31$ to 0.97 ) at age 6.5 years after adjusting for potential confounders. Childhood adherence to a Mediterranean diet was negatively associated with persistent wheeze and atopy although the associations did not reach statistical significance.

Conclusion: These results support a protective effect of a high level of adherence to a Mediterranean diet during pregnancy against asthma-like symptoms and atopy in childhood.

Although considerable advances in knowledge have been gained with studies focused on single nutrients or food items, these may fail to account for the interactions between nutrients, and they do not take into consideration that some nutrients are interrelated. ${ }^{1}$ Thus interest has shifted to the study of food groups and, more recently, dietary patterns that represent a broader picture of food and nutrient consumption and may therefore be more predictive of disease risk. Evaluation of the potential protective role of the Mediterranean diet in respiratory allergic disorders fits well in the context of assessing the overall effect of adherence to a dietary pattern on the occurrence of a disease.
The traditional Mediterranean diet refers to a dietary pattern in the Mediterranean olive grove areas at the beginning of the 1960s. ${ }^{2}$ In general, the Mediterranean diet is characterised by elevated intake of plant foods such as fruits and vegetables, bread and cereals (primarily wholegrain), legumes and nuts. Low to moderate amounts of dairy products and eggs, and only little amounts of red meat are included in the diet. This dietary pattern is low in saturated fatty acids, rich in carbohydrates, fibre and antioxidants, and has a high content of monounsaturated fatty acids and n-3 polyunsaturated fatty acids, which are primarily derived from olive oil and fish intake. ${ }^{3}$

A cross sectional survey in Crete, Greece, indicated that a high level of adherence to a Mediterranean diet during childhood may have a beneficial effect on allergic rhinitis, asthma-like symptoms and atopy, although this was not statistically significant for the last two outcomes. ${ }^{4}$ Another cross sectional study in Spain has shown that a higher adherence to a Mediterranean diet could have a protective effect on current severe asthma in girls aged 6-7 years. ${ }^{5}$ To the best of our knowledge, there are no prospective data on the impact of maternal adherence to a Mediterranean diet during pregnancy on allergy and asthma in their offspring.

In a previous analysis from a birth cohort in Menorca, we found that maternal fish consumption during pregnancy had a protective effect on eczema, atopy and atopic wheeze in the offspring, ${ }^{6}$ while intakes of fish and fruity vegetables in childhood had a beneficial effect on atopy and current wheeze, respectively. ' We used data from the same cohort of women enrolled during pregnancy and whose children were followed-up to 6.5 years of age to test the hypothesis that maternal adherence to a Mediterranean diet during pregnancy and children's adherence to this type of diet have an impact on asthma symptoms and atopy in childhood.

\section{METHODS}

The study methods have been described in detail previously. ${ }^{8}$ Women presenting for antenatal care at all general practices in Menorca ( $n=507,95 \%$ of eligible mothers) over a 12 month period starting in mid-1997 were recruited. A total of 482 children were subsequently enrolled and 468 provided complete outcome data after 6.5 years of followup. Eight children were excluded from the analysis because of implausible values for total energy intake (outside the range of $800-3000 \mathrm{kcal} /$ day). The study was approved by the corresponding 
ethics committees and written informed consent was obtained from the parents of all children.

The outcomes of interest were persistent wheeze at age 6.5 years (described as one or more episodes of "whistling or wheezing from the chest, but not noisy breathing from the nose" over the past 12 months at 6.5 years and in any preceding years), atopic wheeze at age 6.5 years (defined as current wheeze and atopy at this age) and atopy at 6.5 years (based on the skin prick test response).

During the follow-up, parents answered a questionnaire on a yearly basis (with an interviewer) and reported all medical events over the preceding 12 months. Information on parental education, socioeconomic background (using the UK Registrar General's 1990 classification according to parental occupation by ISCO88 code), marital status, maternal disease, parity and children's cigarette exposures (during the mother's pregnancy and at the child's age of 6.5 years) was obtained through questionnaires administered at pregnancy and at the follow up at 6.5 years. ${ }^{9}$ Child body mass index (BMI) at 6.5 years was calculated from weight and height measured with the same instrument in all subjects using a standardised protocol. Weight and height measurements were available for 415 children (90.2\%). Children with a BMI at or above the age and sex specific 85th BMI percentile were classified as overweight whereas those above the 95th BMI percentile were classified as obese. ${ }^{10}{ }^{11}$

\section{Skin prick tests}

A total of 412 children (89.6\%) underwent skin prick testing at 6.5 years of age using a series of six common aeroallergens. ${ }^{7}$ Maternal atopy to common aeroallergens (Dermatophagoides pteronyssinus, Cladosporium, grass pollen, tree mix, graminae mix, cat epithelium) was measured by skin prick tests performed 6 months after delivery. ${ }^{12}$

\section{Assessment of diet}

A semiquantitative food frequency questionnaire (FFO) of 96 food items was used to assess usual dietary intake in children at 6.5 years. ${ }^{7}$ For each participant, the usual daily intake of each food and food groups was estimated in grams per day based on standard portion sizes. Total energy intake was estimated using nutrient composition from the foods composition tables of the US Department of Agriculture (http://www.nal.usda.gov/fnic/ foodcomp/search).

Maternal dietary habits during pregnancy were measured with a shorter (some foods were regrouped into food groups) Spanish version of the validated EPIC-Norfolk FFQ. ${ }^{13}$ All mothers completed a 42 item FFO. The number of times participants consumed specific foods during pregnancy was classified into never, times per year, monthly, weekly or daily depending on the frequencies reported assuming a standard portion size for each item.

The degree of children's adherence to a traditional Mediterranean diet was based on the KidMed index, a Mediterranean diet quality index constructed to evaluate food habits in a population of children. ${ }^{14}$ In the present study, we made minor adaptations to the index to make it relevant to our study population; thus we excluded two questions regarding breakfast intake (skipping breakfast, intake of baked foods or pastries for breakfast). Furthermore, we did not have the information on whether fast foods (hamburgers) were prepared at home or at a fast food restaurant. Consumption of dietary compounds positively associated with a Mediterranean diet (vegetables, legumes, fruits, nuts, cereal, fish, dairy products, olive oil) was assigned a value of +1 whereas intake of compounds with a negative association (sweets, fast foods) was assigned a value of -1 . In the present study, the index ranged from -2 to 12 , and was categorised into three groups: $(1) \geqslant 7$, optimal Mediterranean diet; (2) 4-6, medium quality Mediterranean diet; $(3) \leqslant 3$, low quality Mediterranean diet.

To evaluate adherence to a Mediterranean diet during pregnancy, we used a scale based on the "Mediterranean Diet Score" applied to a large cohort study (EPIC) in adults. ${ }^{15}$ For beneficial components (vegetables, legumes, fruits and nuts, cereal, fish, dairy products), women whose consumption was below the median were assigned a value of 0 and women whose consumption was at or above the median were assigned a value of 1. For components presumed to be detrimental (meat), women whose consumption was below the median were assigned a value of 1 and women whose consumption was at or above the median were assigned a value of $0 .{ }^{15}$ Because the index had been developed for adults and our study population involved pregnant women, we presumed diary products to be protective and not detrimental dietary compounds, and did not include alcohol consumption in the index. The total Mediterranean Diet Score ranged from 0 (minimal adherence to the traditional Mediterranean diet) to 7 (maximal adherence). The score was divided into two levels: (1) 4-7, high Mediterranean diet quality; $(2) \leqslant 3$, low Mediterranean diet quality.

\section{Statistical analysis}

Data analysis was performed using SPSS 13.0 (SPSS Inc, Chicago, Illinois, USA). The primary outcome variables of interest were persistent wheeze, atopic wheeze and atopy at 6.5 years (based on the skin prick test response). As children with atopy and children with wheeze may differ, we created a four category wheeze-atopy status variable (both wheeze and atopy, wheeze according to the questionnaire alone, atopy alone, and neither wheeze nor atopy).

The primary exposures of interest were adherence to a Mediterranean diet for the children (KidMed Score) and the mothers (Mediterranean Diet Score) during pregnancy. Multivariate logistic regression models were further performed to examine the association between adherence to a Mediterranean diet (of the children and mothers separately) and the outcomes of interest after adjusting for confounders. The following variables were considered as potential confounding factors: gender, maternal age at pregnancy, maternal and paternal asthma, maternal atopy, maternal social class and education, maternal smoking during pregnancy, supplement use during pregnancy, breastfeeding, lower track respiratory infections at age 1 year, birth weight, gestational age, birth order, number of siblings and BMI at age 6.5 years. Variables related to the outcomes of interest in the bivariate models with a $p$ value $<0.2$ were included in the multivariate models. All analyses were adjusted for total energy intake using the standard multivariate method. ${ }^{16}$ Odds ratios (OR) and 95\% confidence intervals $(95 \% \mathrm{CI})$ were computed to estimate the degree of association. To test for linear trend (Wald test), the KidMed score was included as an ordinal variable in the logistic model. All hypothesis testing was conducted assuming a 0.05 significance level and a two sided alternative hypothesis.

\section{RESULTS}

The characteristics of the mothers and children who participated in the study in relation to the outcomes of interest are 
Table 1 Adherence to a Mediterranean diet according to the KidMed index* in Menorcan children at age 6.5 years

\begin{tabular}{|c|c|c|c|c|c|c|c|}
\hline & & \multicolumn{2}{|c|}{$\begin{array}{l}\text { All children } \\
(\mathrm{n}=460)\end{array}$} & \multicolumn{2}{|c|}{$\begin{array}{l}\text { Females } \\
(n=228)\end{array}$} & \multicolumn{2}{|c|}{$\begin{array}{l}\text { Males } \\
(n=232)\end{array}$} \\
\hline & & $\mathrm{n}$ & $(\%)$ & $\mathrm{n}$ & $(\%)$ & $\mathrm{n}$ & $(\%)$ \\
\hline \multicolumn{8}{|l|}{ Mediterranean diet components } \\
\hline Fruit or fruit juice daily & +1 & 398 & $(86.5)$ & 193 & (84.7) & 205 & (88.4) \\
\hline Second serving of fruit daily & +1 & 201 & (43.7) & 98 & (43.0) & 103 & (44.4) \\
\hline Fresh or cooked vegetables daily & +1 & 228 & (49.6) & 109 & (47.8) & 119 & (51.3) \\
\hline Fresh or cooked vegetables $>1 /$ day & +1 & 24 & (5.2) & 9 & $(4.0)$ & 15 & (6.5) \\
\hline Legumes $\geqslant 1 /$ week & +1 & 406 & $(88.3)$ & 197 & (86.4) & 209 & (90.1) \\
\hline Regular fish consumption (at least $2-3 /$ week) & +1 & 381 & (82.8) & 188 & (82.5) & 193 & (83.2) \\
\hline Cereals for breakfast $\geqslant 1 /$ day & +1 & 86 & (18.7) & 37 & $(16.2)$ & 49 & (21.1) \\
\hline Pasta or rice almost daily ( $\geqslant 5 /$ week) & +1 & 274 & (59.6) & 129 & (56.6) & 145 & (62.5) \\
\hline Dairy product (milk, yogurt or cheese) daily & +1 & 459 & (99.8) & 228 & (99.6) & 231 & (99.6) \\
\hline Two dairy products daily & +1 & 433 & (94.1) & 211 & (92.5) & 222 & (95.7) \\
\hline Regular nut consumption (2-3/week) & +1 & 77 & $(16.7)$ & 41 & $(18.0)$ & 36 & (15.5) \\
\hline Use of olive oil at home daily & +1 & 347 & (75.4) & 173 & (75.9) & 174 & (75.0) \\
\hline Fast food consumption (hamburger) $>1 /$ week & -1 & 157 & $(34.1)$ & 72 & (31.6) & 85 & (36.6) \\
\hline Sweets-pastries $\geqslant 1 /$ day & -1 & 450 & (97.8) & 225 & (98.7) & 225 & (97.0) \\
\hline \multicolumn{8}{|l|}{ KidMed Score } \\
\hline Low $(\leqslant 3)$ & & 43 & (9.3) & 23 & $(10.1)$ & 20 & (8.6) \\
\hline Medium (4-6) & & 247 & (53.7) & 122 & (53.5) & 125 & (53.9) \\
\hline High $(\geqslant 7)$ & & 170 & $(37.0)$ & 83 & (36.4) & 87 & (37.5) \\
\hline
\end{tabular}

shown in table S1 (online supplement). The prevalence rates of persistent wheeze, atopic wheeze and atopy at age 6.5 years were $13.2 \%, 5.8 \%$ and $17.0 \%$ respectively.

Table 1 presents the degree of adherence to a Mediterranean diet according to the KidMed Index among children at 6.5 years of age. A low index was found for $9.3 \%$ of the sample, $53.7 \%$ had intermediate values and $37.0 \%$ a high index. Multivariate logistic regression analysis showed negative associations, although not statistically significant, between a high level of adherence to a Mediterranean diet and persistent wheeze, atopic wheeze and atopy at age 6.5 years (table 2).

One-third $(166,36.1 \%$ ) of the mothers had a low quality Mediterranean diet during pregnancy according to the Mediterranean Diet Score, while the rest had a high score. Associations between maternal intake of different food groups included in the Mediterranean diet during pregnancy with wheeze and atopy in children at age 6.5 years are presented in table 3. Consumption of vegetables more than eight times per week was inversely associated with persistent wheeze (OR 0.36; $95 \%$ CI 0.14 to 0.92 ) and atopy (OR $0.40 ; 95 \%$ CI 0.22 to 0.72 ). Cereal intake showed a trend towards negative associations with persistent and atopic wheeze, although this was not statistically significant. Fish intake more than 2-3 times per week and intake of legumes more than once per week were inversely associated with persistent wheeze (OR 0.34, 95\% CI 0.13 to 0.84 ; OR $0.36,95 \%$ CI 0.13 to 1.01 , respectively). In contrast, an increased intake of red meat (more than 3-4 times per week) showed a trend towards positive associations with persistent wheeze and atopic wheeze in the offspring (table 3).

Table 4 presents the association of maternal adherence to a Mediterranean diet during pregnancy with wheeze and atopy in children at 6.5 years of age. A high Mediterranean Diet Score was found to be protective for persistent wheeze, atopic wheeze and atopy at 6.5 years of age. Maternal Mediterranean diet during pregnancy was highly associated with adherence to this type of diet during childhood $\left(\chi^{2}(\mathrm{df} 2)=12.92, \mathrm{p}=0.002\right)$ but the two dietary scores did not interact with each other significantly. When we simultaneously included maternal and children Mediterranean Diet Index in the multivariate models, the results remained very similar, showing an independent beneficial effect of maternal diet during pregnancy on wheeze and atopy at 6.5 years of age (table 4 ).

Analysis by child-mother Mediterranean diet status at four levels showed that a high level of maternal adherence to a Mediterranean diet during pregnancy (Mediterranean Diet score $>3$ ) among children with a low KidMed score $(\leqslant 6)$ had a

Table 2 Association of children's adherence to a Mediterranean diet with wheeze and atopy in Menorcan children at age 6.5 years

\begin{tabular}{|c|c|c|c|}
\hline & \multicolumn{2}{|l|}{ KidMed Score } & \multirow[b]{2}{*}{$\begin{array}{l}\text { p Value } \\
\text { for trend }\end{array}$} \\
\hline & $\begin{array}{l}\text { Medium (4-6) } \\
\text { OR (95\% CI) }\end{array}$ & $\begin{array}{l}\text { High }(\geqslant 7) \\
\text { OR }(95 \% \mathrm{CI})\end{array}$ & \\
\hline Persistent wheeze at 6.5 years* $(n=37)$ & $0.44(0.10-1.91)$ & $0.46(0.10-2.17)$ & 0.523 \\
\hline Atopic wheeze at 6.5 years $\uparrow(n=20)$ & $0.51(0.09-2.89)$ & $0.64(0.10-4.06)$ & 0.902 \\
\hline Atopy $\dagger(n=70)$ & $0.58(0.23-1.45)$ & $0.49(0.18-1.32)$ & 0.213 \\
\hline
\end{tabular}


Table 3 Associations between maternal food intake during pregnancy with wheeze and atopy in Menorcan children at age 6.5 years

\begin{tabular}{|c|c|c|c|c|c|c|c|}
\hline \multirow{3}{*}{$\begin{array}{l}\text { Maternal food intake } \\
\text { during pregnancy }\end{array}$} & \multirow{3}{*}{$\begin{array}{l}\text { Median } \\
\text { (servings per } \\
\text { week) }\end{array}$} & \multicolumn{6}{|c|}{ Prevalence $(\%)$ of the outcomes at age 6.5 years } \\
\hline & & \multicolumn{2}{|c|}{$\begin{array}{l}\text { Persistent wheeze } \dagger \\
(\mathrm{n}=37)\end{array}$} & \multicolumn{2}{|c|}{$\begin{array}{l}\text { Atopy: } \\
(n=70)\end{array}$} & \multicolumn{2}{|c|}{$\begin{array}{l}\text { Atopic wheeze } \\
(\mathrm{n}=20)\end{array}$} \\
\hline & & n & $(\%)$ & $\bar{n}$ & $(\%)$ & $n$ & $(\%)$ \\
\hline \multicolumn{8}{|c|}{ Mediterranean diet components } \\
\hline \multirow[t]{2}{*}{ Cereals } & $\leqslant 11.5$ & 21 & 15.22 & 34 & 17.00 & 14 & 8.14 \\
\hline & $>11.5$ & 16 & 11.27 & 36 & 17.06 & 6 & 3.51 \\
\hline \multirow[t]{2}{*}{ Fruits and nuts } & $\leqslant 15$ & 16 & 12.50 & 37 & 17.87 & 10 & 5.85 \\
\hline & $>15$ & 21 & 13.82 & 33 & 16.18 & 10 & 5.81 \\
\hline \multirow[t]{2}{*}{ Fruits } & $\leqslant 14$ & 22 & 13.58 & 45 & 17.65 & 13 & 6.13 \\
\hline & $>14$ & 15 & 12.82 & 25 & 16.23 & 7 & 5.43 \\
\hline \multirow[t]{2}{*}{ Nuts } & $\leqslant 1$ & 19 & 12.50 & 37 & 16.09 & 9 & 4.71 \\
\hline & $>1$ & 18 & 14.06 & 33 & 18.23 & 11 & 7.24 \\
\hline \multirow[t]{2}{*}{ Legumes } & $\leqslant 1$ & 26 & 14.69 & 49 & 18.35 & 15 & 6.79 \\
\hline & $>1$ & 11 & $10.68^{*}$ & 21 & 14.58 & 5 & 4.10 \\
\hline \multirow[t]{2}{*}{ Vegetables } & $\leqslant 8$ & 22 & 15.17 & 47 & 21.46 & 13 & 7.39 \\
\hline & $>8$ & 15 & $11.11^{*}$ & 23 & $11.98 * *$ & 7 & 4.19 \\
\hline \multirow[t]{2}{*}{ Fish } & $\leqslant 2.5$ & 24 & 17.02 & 36 & 16.59 & 13 & 7.10 \\
\hline & $>2.5$ & 13 & $9.35^{*}$ & 34 & 17.53 & 7 & 4.38 \\
\hline \multirow[t]{2}{*}{ Red meat } & $\geqslant 3.25$ & 17 & 16.50 & 23 & 16.31 & 9 & 7.63 \\
\hline & $<3.25$ & 20 & 11.30 & 47 & 17.41 & 11 & 4.89 \\
\hline \multirow[t]{2}{*}{ White meat } & $\geqslant 2.5$ & 10 & 12.50 & 21 & 17.65 & 7 & 7.00 \\
\hline & $<2.5$ & 27 & 13.50 & 49 & 16.78 & 13 & 5.35 \\
\hline \multirow[t]{2}{*}{ Dairy products } & $\leqslant 23$ & 18 & 14.06 & 34 & 16.83 & 12 & 6.90 \\
\hline & $>23$ & 19 & 12.50 & 36 & 17.22 & 8 & 4.73 \\
\hline
\end{tabular}

All models were adjusted for gender, maternal and paternal asthma, maternal social class and education, body mass index and total energy intake at age 6.5 years.

Also adjusted for: ffirstborn and lower respiratory track infections at age 1; tbirth weight and maternal atopy.

$\mathrm{n}$, number of cases; \%, prevalence of outcome.

${ }^{*} \mathrm{p}<0.05$, ${ }^{* *} \mathrm{p}<0.01$.

protective effect on persistent wheeze at age 6.5 years (OR 0.20; $95 \%$ CI 0.06 to 0.69 ) while in children with a high KidMed score $(>6)$ there was also a protective effect although this was only marginally significant (OR $0.29 ; 95 \%$ CI 0.09 to 1.01 ). Children who had a high KidMed score at age 6.5 years and their mothers had a high Mediterranean Diet Score in pregnancy were also found to be protected against atopy (OR $0.44 ; 95 \%$ CI 0.19 to 1.02). Regarding atopic wheeze, there was a trend for a protective effect of a high Mediterranean Diet Score in pregnancy, although the associations were not significant, possibly because of the small numbers (fig 1).

\section{DISCUSSION}

In this prospective cohort study, we observed a reduced risk of wheeze and atopy among children at age 6.5 years whose

Table 4 Association of maternal adherence to a Mediterranean diet during pregnancy with wheeze and atopy in Menorcan children at age 6.5 years

\begin{tabular}{|c|c|c|}
\hline & \multicolumn{2}{|c|}{ Maternal high Med Diet Score } \\
\hline & OR $(95 \%$ CI) & OR: $(95 \% \mathrm{CI})$ \\
\hline Persistent wheeze at 6.5 years $^{*}(n=37)$ & $0.23(0.09-0.60)$ & $0.22(0.08-0.58)$ \\
\hline Atopic wheeze at 6.5 years $\dagger(n=20)$ & $0.34(0.12-0.97)$ & $0.30(0.10-0.90)$ \\
\hline Atopy $(\mathrm{n}=70)$ & $0.55(0.32-0.97)$ & $0.55(0.31-0.97)$ \\
\hline
\end{tabular}

All models were adjusted for gender, maternal and paternal asthma, maternal social class and education, body mass index and total energy intake at age 6.5 years. The reference group included those children whose mothers had a low level of adherence to a Mediterranean diet during pregnancy.

Also adjusted for: *firstborn and lower respiratory track infections at age 1; tbirth weight and maternal atopy.

$\$ \mathrm{OR}$, children's adherence to a Mediterranean diet also included in the model. mothers had a high adherence to a Mediterranean diet during pregnancy. To our knowledge, this is the first longitudinal study to assess prospectively in a general population the impact of maternal adherence to a Mediterranean diet during pregnancy and children's adherence to this type of diet on asthma and atopy outcomes in childhood.

Epidemiological and immunological studies suggest that dietary modification or supplementation in fetal life can reduce the development of atopic diseases while fetal undernutrition can detrimentally affect the "programming" of the fetal lung and immune system. ${ }^{17-19}$ The ALSPAC birth cohort study in the UK has shown that umbilical cord concentration of selenium was negatively associated with wheezing, cord concentration of iron was negatively associated with wheezing and eczema in early childhood, while fetal exposure to $n-6$ and n-3 fatty acids was not found to be significantly associated with the outcomes of interest. ${ }^{20}{ }^{21}$ Other longitudinal studies have shown that a higher maternal intake of vitamins $\mathrm{E}^{22-24}$ and $\mathrm{D}^{25}{ }^{26}$ during pregnancy may decrease the risk of wheeze symptoms in early childhood. The LISA birth cohort study in Germany suggested that intake of foods rich in $\mathrm{n}-6$ fatty acids during pregnancy may increase and foods rich in $\mathrm{n}-3$ fatty acids may decrease the risk of allergic diseases in the offspring. ${ }^{27} \mathrm{~A}$ recent longitudinal study showed a protective effect of maternal apple and fish intake during pregnancy against the development of asthma and eczema, respectively, in 5-year-old children in Scotland. ${ }^{28}$ Similarly, we have previously shown that maternal fish intake during pregnancy had a protective effect on the risk of eczema at age 1 year and the risk of atopy and atopic wheeze at age 6.5 years. ${ }^{6}$

Dietary patterns such as Mediterranean diet account for cumulative and interactive effects among nutrients, reflect real 
Figure 1 Associations of maternal and children adherence to a Mediterranean diet with wheeze and atopy in Menorcan children at age 6.5 years. $†$ All models were adjusted for: gender, maternal and paternal asthma, maternal social class and education, body mass index and total energy intake at age 6.5 years. (1) Also adjusted for firstborn and lower respiratory track infections at age 1 year; (2) also adjusted for birth weight and maternal atopy.

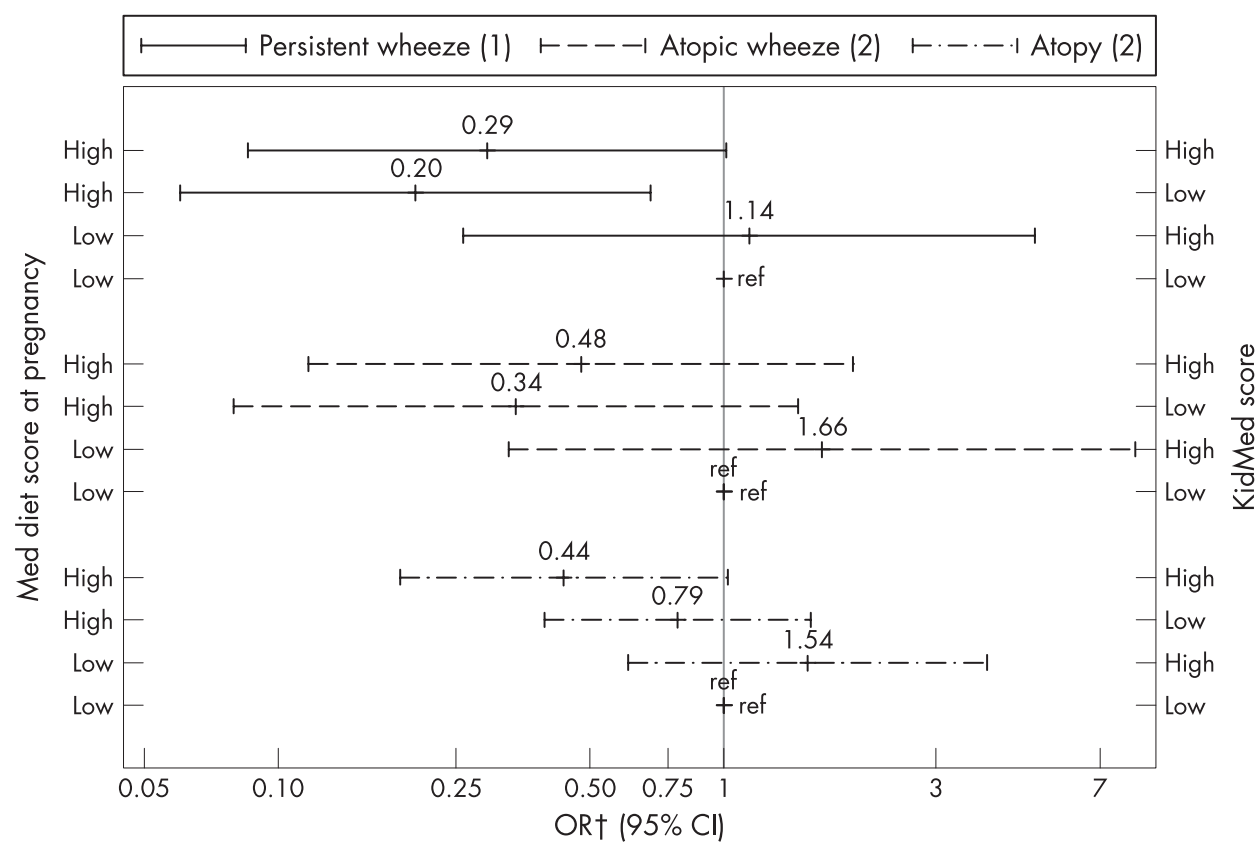

world dietary preferences and may be particularly suitable for analysis in asthma epidemiology where many dietary components could be related to the outcome of interest. ${ }^{29}$ The results of the present study, indicating a protective effect of maternal adherence to a Mediterranean diet during pregnancy on wheeze and atopy at age 6.5 years, probably reflect high fetal exposure to several antioxidant compounds and their adverse effect on oxidative stress damage of lung tissues. Cereals (particularly wholegrains) are rich in antioxidant compounds (ie, vitamin E, phenolic acids and phytic acid) and they have been shown to have a protective effect against asthma in children. ${ }^{30}{ }^{31}$ Similarly, fruits, vegetables and legumes are known to be high sources of antioxidants (vitamins C, E, carotenoids, selenium, flavonoids) and may therefore help to protect the airways against oxidative damage. Apart from the most widely known antioxidants, other compounds, such as oleuropein, hydroxytyrosol and other polyphenols present in olive oil, the principal component of a Mediterranean diet, have marked antioxidant activity and other advantageous biological properties. ${ }^{32}$ On the other hand, the polyunsaturated n-3 fatty acids eicosapentaenoic and docosahexaenoic found in fish oil have anti-inflammatory effects, and a high intake during pregnancy has been associated with a reduced risk of allergic diseases in childhood. ${ }^{67} 28$

The time window of exposure is becoming a key aspect in the study of diseases involving systems with a long developmental length such as the immunological and respiratory systems. ${ }^{33}$ It is possible that the immunomodulating benefits of antioxidant compounds may be greater during critical stages of early immune development before an allergic response is established. Allergen specific responses are already evident at birth and allergic disease is often manifest within the first month of life, suggesting that the processes that lead to allergic diseases can be initiated very early in immune development. ${ }^{6}{ }^{19}$ On the other hand, it is well established that during gestation, essential nutrients are transferred from the maternal to the fetal circulation across the placenta, and such transport mechanisms have been identified for antioxidants and long chain polyunsaturated fatty acids, basic principles of the Mediterranean diet. ${ }^{34}$ As a result, the protective effect of a Mediterranean diet on the development of asthma and allergies could be higher during pregnancy than during childhood. This is consistent with our results that indicate a stronger and independent effect of maternal adherence to a Mediterranean diet during pregnancy on persistent wheeze and atopy rather than childhood diet. Childhood adherence to a Mediterranean diet was negatively associated with persistent wheeze although the association was not statistically significant. When we analysed foods separately, we found a protective effect of fruity vegetables and fish intake during childhood on wheeze and atopy, respectively?

Adherence to a Mediterranean diet during pregnancy was evaluated according to the Mediterranean Diet Score, a validated index widely used for evaluation of adherence to a Mediterranean diet in adults. ${ }^{15}$ As this is the first time that maternal adherence to a Mediterranean diet in pregnancy has been evaluated in relation to disease outcome in childhood, some questions could be raised as to the effectiveness of the Mediterranean diet in pregnancy in relation to increased maternal nutritional needs and to fetal outcome. Firstly, several studies have demonstrated that, apart from better dietary fat quality and increased quantity of antioxidants, the high nutritional quality of the Mediterranean diet is devoid of risks of inadequate intake of several vitamins and minerals. . $^{36}$ Because alcohol consumption is prohibited in pregnancy, and during this period there are increased needs for calcium intake, we did not include in the index alcohol consumption and we presumed diary compounds to be protective and not detrimental. Moreover, clinical outcome studies suggest that bone mineral mass in newborn infants is related to maternal size and dairy intake during pregnancy. ${ }^{37}$ We found no significant associations between a high level of adherence to a Mediterranean diet during pregnancy and gestational age $(p=0.477)$ or birth weight $(p=0.906)$.

In this analysis, fruits and nuts were grouped together as this was the initial categorisation used in the Mediterranean Diet Score ${ }^{15}$ Intake of nuts during pregnancy in our population was relatively low, it was not associated with maternal asthma $(p=0.106)$ or maternal atopy $(p=0.506)$ while it showed an association with a somewhat higher prevalence of wheeze and atopy in children, although this was not statistically significant. 
A total of 101 women (22\%) excluded nuts from their diet during pregnancy and of them, 96\% (97) ate at least one piece of fruit per week. The distribution of the Mediterranean Diet Score was quite similar in mothers who excluded nuts and fruits from their diet and in mothers who excluded only nuts (data not shown). However, we have no information on maternal food allergy and therefore we cannot exclude the possibility of exclusion of nuts from the diet because of food allergy.

The strengths of the present study include the population based design, reasonable numbers of exposed children and fairly detailed data for dietary intake for both mothers and children. Unlike previous epidemiological studies on diet and asthma in children, adherence to a Mediterranean diet was adjusted for total energy intake which allowed us to control for unknown confounders and better address whether dietary reporting might have been biased (eg, by identifying energy under/overreporters). ${ }^{19}$ Furthermore, the study population included children from the follow-up of a birth cohort, giving us the opportunity to account for the effect of exposures during pregnancy and early life measured prospectively within the cohort. We did not observe any substantial differences between the crude and adjusted models. Thus it is unlikely that overadjustment affected our findings.

We have considered parental reports on children's diet and symptoms, and information bias could have occurred. However, studies of nutrition in childhood suggest that food frequency methods produce both valid and reproducible estimates of the dietary intakes of children and adolescents. ${ }^{38}{ }^{39}$ Indexes such as the KidMed Index and Mediterranean Diet Score that use scorings have certain limitations such as the variability in choosing cut-off points in the score and the different distribution of selected food groups in different populations. ${ }^{40} \mathrm{~A}$ sensitivity analysis using Mediterranean Diet Score in pregnancy as a continuous variable gave essentially the same results as the categorical analysis, indicating a protective effect per unit increase for persistent wheeze (OR 0.69; $95 \%$ CI 0.50 to 0.97 ) and atopic wheeze (OR $0.79 ; 95 \%$ CI 0.55 to 1.13 ) while for atopy there was no strong protective effect (OR $0.93 ; 95 \% \mathrm{CI} 0.76$ to 1.13) (data not shown). Participants were unaware of the Mediterranean diet hypothesis being tested and therefore misclassification of intake estimated by the FFQ is likely to be random with respect to wheeze and atopy. Persistent wheeze at age 6.5 years was based on the prospective evaluation of symptoms since birth reported by the mother. Wheeze at age 6.5 years is less associated with low respiratory infections than at an earlier age and therefore more likely to be a marker of asthma.

In conclusion, the present study suggests that a high level of adherence to a Mediterranean diet during pregnancy may protect against asthma-like symptoms and atopy in childhood. Further studies are needed to better understand the mechanisms of this protective effect and the most relevant window of exposure. Further follow-up of this cohort will allow determining if this protective effect persists in older children.

Funding: The study was supported by the Instituto de Salud Carlos III red de Grupos Infancia y Media Ambiente (G03/176), the Fundacio "La Caixa" (00/077-00), the Instituto de Salud Carlos III, red de Centros de Investigacion en Epidemiologia y Salud Publica (C03/09) and EU grant FOOD-CT-2005-016320 NewGeneris. Dr Romieu was supported in part by the National Center for Environmental Health - Centers for Disease Control and Prevention, Atlanta, Georgia, USA, the GA2LEN project (EU contract FOODCT-2004-506378) and the Ministry of Education and Science, Spain, SAB20040192. Dr Vioque was additionally supported by the Oficina de Ciencia y Tecnología, Generalitat Valenciana (grant CTGCA/2002/06 G03/136).

Competing interests: None.

Ethics approval: Ethical approval was obtained.

\section{REFERENCES}

1. Jacobs DR Jr, Steffen LM. Nutrients, foods, and dietary patterns as exposures in research: a framework for food synergy. Am J Clin Nutr 2003;78:508-13.

2. Willett WC, Sacks F, Trichopoulou A, et al. Mediterranean diet pyramid: a cultural model for healthy eating. Am J Clin Nutr 1995;61(Suppl):1402-6.

3. Trichopoulou A, Lagiou P. Healthy traditional Mediterranean diet: an expression of culture, history, and lifestyle. Nutr Rev 1997;55:383-9.

4. Chatzi L, Apostolaki G, Bibakis I, et al. Protective effect of fruits, vegetables and the Mediterranean diet on asthma and allergies among children in Crete. Thorax 2007;62:677-83.

5. Garcia-Marcos L, Miner Canflanca I, Batlles Garrido J, et al. The relationship of asthma and rhinoconjunctivitis with obesity, exercise and Mediterranean diet in Spanish schoolchildren 6-7 years old. Thorax 2007;62:503-8.

6. Romieu I, Torrent M, Garcia-Esteban R, et al. Maternal fish intake during pregnancy and atopy and asthma in infancy. Clin Exp Allergy 2007;37:518-25.

7. Chatzi L, Torrent M, Romieu I, et al. Diet, wheeze, and atopy among school children in Menorca, Spain. Pediatr Allergy Immunol 2007;18:480-5.

8. Sunyer J, Torrent M, Garcia-Esteban R, et al. Early exposure to dichlorodiphenyldichloroethylene, breastfeeding and asthma at age six. Clin Exp Allergy 2006;36:1236-41.

9. Polk S, Sunyer J, Munoz-Ortiz L, et al. A prospective study of Fel d1 and Der p1 exposure in infancy and childhood wheezing. Am J Respir Crit Care Med 2004;170:273-8.

10. Reilly JJ, Wilson ML, Summerbell CD, etal. Obesity: diagnosis, prevention, and treatment; evidence based answers to common questions. Arch Dis Child 2002;86:392-4.

11. Barlow SE, Dietz WH. Obesity evaluation and treatment: Expert Committee recommendations. The Maternal and Child Health Bureau, Health Resources and Services Administration and the Department of Health and Human Services. Pediatrics 1998;102:E29.

12. Sunyer J, Anto JM, Harris J, et al. Maternal atopy and parity. Clin Exp Allergy 2001;31:1352-5

13. Bingham SA, Welch AA, McTaggart $A$, et al. Nutritional methods in the European Prospective Investigation of Cancer in Norfolk. Public Health Nutr 2001;4:847-58.

14. Serra-Majem L, Ribas L, Ngo J, et al. Food, youth and the Mediterranean diet in Spain. Development of KIDMED, Mediterranean Diet Quality Index in children and adolescents. Public Health Nutr 2004;7:931-5.

15. Trichopoulou A, Costacou T, Bamia C, et al. Adherence to a Mediterranean diet and survival in a Greek population. N Engl J Med 2003;348:2599-608.

16. Willet WC, Stampfer MJ. Total energy intake: implications for epidemiologic analyses. Am J Epidemiol 1986;124:17-27.

17. Langley-Evans S. Fetal programming of immune function and respiratory disease. Clin Exp Allergy 1997;27:1377-9.

18. Devereux G, Seaton A. Diet as a risk factor for atopy and asthma. J Allergy Clin Immunol 2005;115:1109-17.

19. Tricon S, Willers S, Smit HA, et al. Nutrition and allergic disease. Clin Exp Allergy Reviews 2006;6:117-88.

20. Shaheen SO, Newson RB, Henderson AJ, et al. Umbilical cord trace elements and minerals and risk of early childhood wheezing and eczema. Eur Respir J 2004;24:292-7.

21. Newson RB, Shaheen SO, Henderson AJ, et al. Umbilical cord and maternal blood red cell fatty acids and early childhood wheezing and eczema. J Allergy Clin Immunol 2004;114:531-7.

22. Martindale S, McNeill G, Devereux G, et al. Antioxidant intake in pregnancy in relation to wheeze and eczema in the first two years of life. Am J Respir Crit Care Med 2005;171:121-8

23. Litonjua AA, Rifas-Shiman SL, Ly NP, et al. Maternal antioxidant intake in pregnancy and wheezing illnesses in children at 2 y of age. Am J Clin Nutr 2006;84:903-11.

24. Devereux G, Turner SW, Craig LC, et al. Low maternal vitamin E intake during pregnancy is associated with asthma in 5-year-old children. Am J Respir Crit Care Med 2006;174:499-507.

25. Camargo CA Jr, Rifas-Shiman SL, Litonjua AA, et al. Maternal intake of vitamin D during pregnancy and risk of recurrent wheeze in children at 3 y of age. Am J Clin Nutr 2007;85:788-95.

26. Devereux G, Litonjua AA, Turner SW, et al. Maternal vitamin D intake during pregnancy and early childhood wheezing. Am J Clin Nutr 2007;85:853-9.

27. Sausenthaler S, Koletzko S, Schaaf B, et al. Maternal diet during pregnancy in relation to eczema and allergic sensitization in the offspring at 2 y of age. Am J Clin Nutr 2007;85:530-7.

28. Willers S, Devereux G, Craig L, et al. Maternal food consumption during pregnancy and asthma, respiratory and atopic symptoms in 5-year-old children. Thorax 2007:62:773-9.

29. Hu FB. Dietary pattern analysis: a new direction in nutritional epidemiology. Curr Opin Lipidol 2002;13:3-9.

30. Wijga AH, Smit HA, Kerkhof M, et al. Association of consumption of products containing milk fat with reduced asthma risk in pre-school children: the PIAMA birth cohort study. Thorax 2003;58:567-72.

31. Tabak C, Wijga AH, de Meer G, et al. Diet and asthma in Dutch school children (ISAAC-2). Thorax 2006;61:1048-53.

32. Fito $\mathbf{M}$, Cladellas $\mathbf{M}$, de la Torre $\mathrm{R}$, et al. Antioxidant effect of virgin olive oil in patients with stable coronary heart disease: a randomized, crossover, controlled, clinical trial. Atherosclerosis 2005;181:149-58.

33. Sunyer J, Torrent M, Munoz-Ortiz L, et al. Prenatal dichlorodiphenyldichloroethylene (DDE) and asthma in children. Environ Health Perspect 2005;113:1787-90. 
34. Schenker S, Yang Y, Perez A, et al. Antioxidant transport by the human placenta. Clin Nutr 1998;17:159-67.

35. Fletcher RH, Fairfield KM. Vitamins for chronic disease prevention in adults: clinical applications. JAMA 2002:287:3127-9.

36. Serra-Majem L, Ribas L, Garcia A, et al. Nutrient adequacy and Mediterranean diet in Spanish school children and adolescents. Eur J Clin Nutr 2003;57(Suppl 1):S35-9.

37. Abrams SA. In utero physiology: role in nutrient delivery and fetal development for calcium, phosphorus, and vitamin D. Am J Clin Nutr 2007;85:604S-7.
38. Rockett HR, Wolf AM, Colditz GA. Development and reproducibility of a food frequency questionnaire to assess diets of older children and adolescents. J Am Diet Assoc 1995;95:336-40.

39. McPherson RS, Hoelscher DM, Alexander M, et al. Dietary assessment methods among school-aged children: validity and reliability. Prev Med 2000;31:S11-33.

40. Bach A, Serra-Majem L, Carrasco JL, et al. The use of indexes evaluating the adherence to the Mediterranean diet in epidemiological studies: a review. Public Health Nutr 2006;9:132-46.

\section{Lung alert}

\section{Diesel exhaust adversely affects people with moderate asthma}

Road traffic pollutions may trigger asthma symptoms and exacerbations, and this is mainly attributed to polycyclic aromatic hydrocarbon core diesel exhaust particles (DEP).

The authors carried out a randomised crossover study enrolling 60 non-smoking adults with stable mild and moderate asthma. Each participant was required to walk for 2 hours (10.3012.30 hours) along Oxford Street, London (used by only diesel powered buses and taxis) and traffic-free Hyde Park during weekdays only between November and March to avoid the pollen season. Both forced expiratory volume in $1 \mathrm{~s}\left(\mathrm{FEV}_{1}\right)$ and forced vital capacity (FVC) were measured immediately before and each hour during the walk and various measurements were obtained for the following $12 \mathrm{~h}$. Concentrations of ultrafine particles, nitrogen dioxide and elemental carbon were measured.

This study showed asymptomatic but consistent spirometric reductions ( $\mathrm{FEV}_{1}$ up to $6.1 \%$ and FVC up to $5.4 \%$ ) in patients with moderate asthma after walking along Oxford Street. These changes were associated with an increase in markers of neutrophilic inflammation (sputum myeloperoxidase and the $\mathrm{pH}$ of their exhaled breath condensate). Participants had significantly higher exposures to ultrafine particles, nitrogen dioxide and elemental carbon on Oxford Street than in Hyde Park, suggesting that DEP may affect airway inflammation in asthma.

The authors point out that the inevitably unblinded nature of the study and the effect of other factors such as noise and stress in a busy road may be confounding factors in this study, but further study is warranted on the basis of these results. They do not currently recommend that people with asthma stop visiting or working in busy urban environments.

- McCreanor J, Cullinan P, Nieuwenhuijsen MJ, et al. Respiratory effects of exposure to diesel traffic in persons with asthma. $N$ Engl $J$ Med 2007;357:2348-58.

\section{Tasbirul Islam}

Correspondence to: Dr T Islam, Department of Pulmonary, Critical Care and Sleep Medicine, Winthrop University Hospital, Mineola, NY 11501, USA; tasbirul@msn.com 